\title{
Azimuthally small-scale Alfvén waves in magnetosphere excited by the source of finite duration
}

\author{
Dmitri Yu. Klimushkin ${ }^{1,3}$, Ivan Yu. Podshibyakin ${ }^{1}$, and Jin Bin $\mathrm{Cao}^{2,3}$ \\ ${ }^{1}$ Institute of Solar-Terrestrial Physics (ISTP), Russian Academy of Science, Siberian Branch, Irkutsk, P.O.Box 291, 664033, Russia \\ ${ }^{2}$ Key Laboratory of Space Weather, Center for Space Science and Applied Research, 100080, Bejing, China \\ ${ }^{3}$ Russian-Chinese Joint Research Center on Space Weather
}

(Received July 13, 2006; Accepted May 21, 2007; Online published August 31, 2007)

\begin{abstract}
In this paper the spatial structure of azimuthally small-scale Alfvén waves in magnetosphere excited by the impulse source is studied. The source is suddenly switched on at a definite moment and works as $e^{-i \omega_{0} t}$ during the finite time interval. The influence of factors which lead to the difference of toroidal and poloidal eigenfrequencies (like curvature of field lines and finite plasma pressure) is taken into account. Due to these factors, a radial component of the group velocity of Alfvén wave appears. An important value is the time moment, $t_{0}$, when a wave front moving with radial component of wave group velocity from the poloidal surface (a magnetic surface where the source frequency $\omega_{0}$ coincides with the poloidal frequency) passes the given magnetic shell with the radial coordinate $x$. The temporal evolution at all the points, where the front has not come yet, is determined by the phase mixing of the initial disturbance. At the points through which the wave front has already passed, the wave field structure almost coincides with the structure of monochromatic wave. The region where the front propagates is bounded by the interval between the poloidal surface and the toroidal one (that is, the Alfvén resonance surface). For this reason, outside this region the evolution is always determined by the phase mixing, which leads to much smaller amplitudes than between poloidal and toroidal surfaces. After the source turned off, a back wave front is formed, which comes through the given point in direction from the poloidal surface to the toroidal one. After the back front has come, the monochromatic wave structure disappears and there is only a weak disturbance, which steadily disappears because of the phase mixing and the final conductivity of ionosphere.
\end{abstract}

Key words: Alfvén wave, poloidal mode, impulse excitation.

\section{Introduction}

Among ultra low-frequency oscillations in the Earth's magnetosphere, the azimuthally small-scale Alfvén waves are distinguished, i.e., the waves with large azimuthal wave numbers $m \gg 1$ (Takahashi, 1988; Anderson, 1993; Denton et al., 2003). These waves usually have poloidal polarization, i.e. field lines oscillate in the radial direction. Theoretical studies of such waves are performed on the basis of magnetosphere models with the field lines curvature, twodimensional inhomogeneity of the plasma and the magnetic field; the plasma finite pressure are taken into account.

The high- $m$ wave sources are supposed to be located inside the magnetosphere (e.g., Takahashi, 1988; Yumoto, 1988). From the mathematical point of view, there are the two limiting cases of the source, a monochromatic one (the spectrum is a delta-function over frequency), and an impulse one (a delta-function over time), in the spectrum of which all the frequencies are equally presented.

Before describing the most important results obtained to date, let us present some definitions. Even in the 1960s it was found out that the purely radial (poloidal) and azimuthal (toroidal) oscillations of field lines were charac-

Copyright (c) The Society of Geomagnetism and Earth, Planetary and Space Sciences (SGEPSS); The Seismological Society of Japan; The Volcanological Society of Japan; The Geodetic Society of Japan; The Japanese Society for Planetary Sciences; TERRAPUB terized by slightly different frequencies (Radoski, 1967; Dungey, 1967), which are designated here as $\Omega_{P N}$ and $\Omega_{T N}$. These frequencies are the functions of a radial coordinate, $x$, which marks the magnetic shells. If the wave frequency $\omega$ is fixed, we can introduce the notions of poloidal $x_{P N}$ and toroidal $x_{T N}$ magnetic shells determined as solutions of the equations $\omega=\Omega_{P N}(x)$ and $\omega=\Omega_{T N}(x)$, respectively.

The theory of monochromatic waves with $m \gg 1$ is rather highly developed at present. Leonovich and Mazur (1993) showed that in a curved magnetic field, the Alfvén waves, being standing waves along the magnetic field, generally propagate across magnetic shells. The region of wave propagation (transparent region) is bounded by the poloidal and toroidal surfaces. The wave is excited near the poloidal surface and propagates toward the toroidal surface where it is totally absorbed due to the dissipation in the ionosphere. During the propagation, the wave polarization changes from poloidal to toroidal (Fig. 1(a)). The width of the transparent region is proportional to the difference between the frequencies of toroidal and poloidal oscillations. The further development of the theory introduced into the general picture such effects as kinetic dispersion (Leonovich and Mazur, 1995a), the finite plasma pressure (Klimushkin, 1998a; Mager and Klimushkin, 2002; Klimushkin et al., 2004), bounce-drift instability (Klimushkin, 2000, 2007), and azimuthal inhomogeneity of magnetosphere (Klimushkin et 

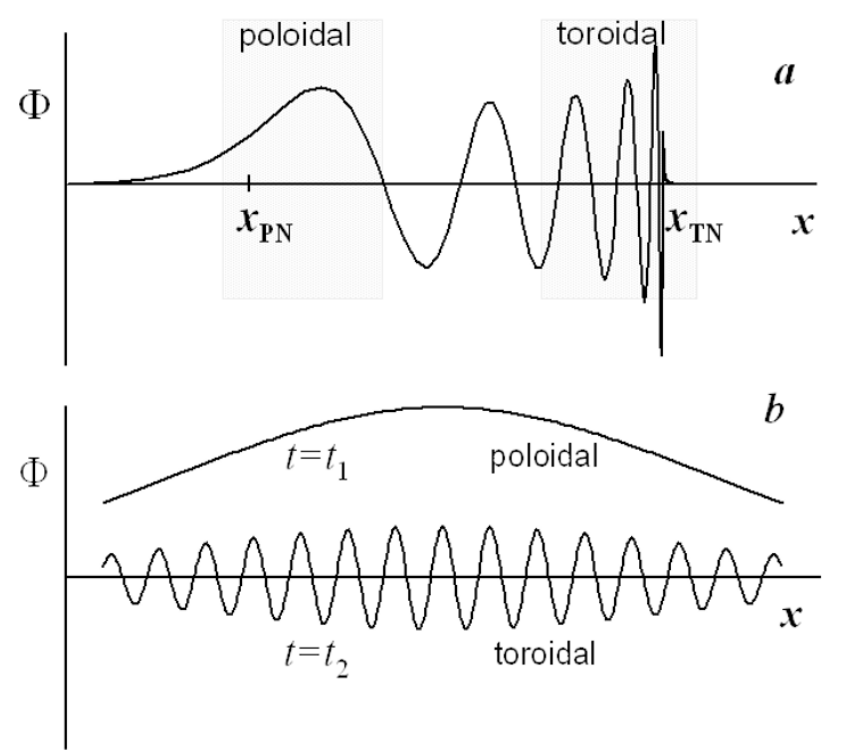

Fig. 1. The transformation of the poloidal Alfvén wave into the toroidal one: (a) in space (monochromatic case), (b) in time (impulse-excited case).

al., 1995). It was also shown that a resonator for the Alfvén wave can exist in the plasmapause and ring current regions where the mode is a standing wave across the magnetic surfaces (Vetoulis and Chen, 1996; Leonovich and Mazur, 1995b; Denton and Vetoulis, 1998; Klimushkin, 1998b; Mager and Klimushkin, 2006).

Let us note that the azimuthal wave number large value itself is not the sufficient condition for the poloidal polarization of monochromatic Alfvén wave. The much stronger condition $\left|\Omega_{T N}-\Omega_{P N}\right| / \Omega_{T N} \gg m^{-1}$ must be satisfied, which implies that many azimuthal wavelengths are accommodated between the toroidal and poloidal surfaces (Klimushkin et al., 2004). By studying several models of the magnetosphere differing in the level of disturbance, Mager and Klimushkin (2002) and Klimushkin et al. (2004) found that this poloidality criterion can be met for the observed values of $m \sim 50-100$ only by taking finite plasma pressure into account.

Opposite to the monochromatic case are the waves generated by the sudden impulse, which is the delta-function of time. In this case, each field line oscillates with its own frequency (Hasegawa et al., 1983); hence the oscillations on neighboring magnetic shells rapidly acquire a significant phase difference (phase mixing), and the wave becomes strongly "indented" in radial coordinate with much smaller amplitude. Thus, the initially poloidally polarized disturbance (sufficiently widely distributed in radial coordinate) transforms into a toroidally polarized wave (Fig. 1(b)). Initially, this was found for the case of onedimensional inhomogeneous model with straight field lines (Radoski, 1974; Mann and Wright, 1995; Mann et al., 1997), but the phase mixing phenomenon was shown to occur also in a dipole-like models (Leonovich and Mazur, 1998; Antonova et al., 1999; Leonovich, 2000). A new feature of impulse-generated high- $m$ Alfvén waves in a curved two-dimensionally inhomogeneous field is a gradual change of the field line oscillations frequency from the local poloidal frequency to the local toroidal frequency, found by Leonovich and Mazur (1998). They gave the following interpretation of this phenomenon: a sudden impulse formally excites a continuous set of monochromatic waves instantaneously on all magnetic shells. On a given magnetic shell, $x$, a monochromatic wave is excited, for which this shell is a poloidal one. Consequently, an oscillation of the poloidal type with the frequency equal to the poloidal frequency of this magnetic shell $x$ will be excited. Correspondingly, the wave's frequency $\omega$ equals the poloidal frequency of this shell $\Omega_{P N}$ in the beginning, and the wave is poloidally polarized. Then, the magnetic surface $x$ is occupied by oscillations arriving from increasingly distant magnetic shells that are originated as poloidal ones there; but as they travel towards this given magnetic surface, they transform progressively to toroidal ones. The drift-bounce resonance with the particles was included in this picture by Klimushkin and Mager (2004). In the course of the propagation, the wave loses its energy because of the finite ionospheric conductivity and acquires energy due to the wave-particle resonant interaction. If the corresponding instability is stronger than the attenuation, then the wave is amplified just after the oscillations have started. But the drift-bounce instability growth rate is getting smaller during the wave temporal evolution, and the instability undergoes stabilization when the wave frequency coincides with the toroidal eigenfrequency.

Of course, both cases, monochromatic and impulse sources, cannot be considered as quite realistic ones. The excitation process in magnetosphere should be nonstationary but have finite time duration. Wright (1992) studied the oscillations generated by the source of this type. He showed that the longer the source is in operation, the narrower the localization of the mode across magnetic shells is. When the duration of the source operation tends to infinity, the mode is concentrated near the field-line resonance surface, as is the case in conventional theory of monochromatic oscillations.

We believe, however, that the results (Wright, 1992) can be directly used only for the azimuthally large-scale oscillations $(m \sim 1)$. In this article, we consider the case $m \gg 1$. The prime aim of the paper is to elucidate how the fine structure of high- $m$ monochromatic wave ripens from initial phase-mixing evolution, and how it disappears after the end of the source operation. Figuratively speaking, we intend to study birth and death of the poloidal Alfvén waves. All the basic results were obtained analytically using various asymptotic methods and illustrated by the graphics obtained numerically.

\section{Radial Structure of the Monochromatic Alfvén Wave}

Within the approximation of ideal plasma conductivity, the longitudinal component of the wave's electric field is zero, i.e. the electric field is a two-dimensional one; it lies on surfaces orthogonal to field lines. As any arbitrary vector field, $\vec{E}_{\perp}$ can split into the sum of potential and vortical components:

$$
\vec{E}=-\nabla_{\perp} \Phi+\nabla_{\perp} \times \vec{e}_{\|} \Psi
$$


where $\vec{e}_{\| \mid}$is a unit vector in the direction of the equilibrium magnetic field. In a homogeneous plasma, the "potentials" $\Phi$ and $\Psi$ describe the electric field of the Alfvén wave and fast magnetosound, respectively (Klimushkin, 1994; Glassmeier, 1995). These MHD modes are described by a system of coupled equations

$$
\begin{gathered}
\hat{L}_{A} \Phi+\hat{L}_{c} \Psi=0 \\
\hat{L}_{F} \Psi+\hat{L}_{c}^{+} \Phi=0,
\end{gathered}
$$

where $\hat{L}_{A}$ and $\hat{L}_{F}$ are differential operators for Alfvén and fast modes, respectively, and operators $\hat{L}_{c}^{+}$and $\hat{L}_{c}$ describe the coupling of these modes (see details in Klimushkin, 1994). The problem is somewhat alleviated in the case $m \gg$ 1 when this system can be brought to a single differential equation describing the Alfvén mode only:

$$
\hat{L}_{A} \Phi=0
$$

(Leonovich and Mazur, 1993). But this equation still contains interrelated problems of determining the longitudinal and transverse structures of the wave. Nonetheless, Vetoulis and Chen (1996), Leonovich and Mazur (1997), and Klimushkin et al. (2004) found that the radial structure of the wave can be qualitatively described by an ordinary differential equation containing derivatives with respect to the coordinate $x$ only, the unit vector of which is directed across magnetic shells:

$$
\begin{aligned}
& \frac{\partial}{\partial x}\left[x-x_{T N}+i a\left(\delta-\gamma \frac{x_{T N}-x}{\Delta_{N}}\right)\right] \frac{\partial \Phi(x, \omega)}{\partial x} \\
& -K^{2}\left[x-x_{P N}+i a\left(\delta-\gamma \frac{x_{T N}-x}{\Delta_{N}}\right)\right] \Phi(x, \omega) \\
& =q(\omega),
\end{aligned}
$$

where $x_{T N}$ and $x_{P N}$ are the coordinates of toroidal and poloidal surfaces (see Introduction), $\Delta_{N}=x_{T N}-x_{P N}$ is a distance between them, $\delta$ is a decrement of the mode damping due to the finite resistance of the ionosphere, the quantity $a \delta \equiv\left|d \Omega_{T N} / d x\right|^{-1} \delta$ determines the typical radial scale associated with the damping (Southwood and Hughes, $1983), \gamma$ is an instability growth rate, caused by the resonant wave-particle interaction, the quantity $K \equiv m / L$ determines the azimuthal component of wave vector ( $m$ is the azimuthal wave number and $L$ is the McIlvein parameter), $q$ is the oscillations source value. It is supposed in Eq. (1) that the functions $\Omega_{P N}(x)$ and $\Omega_{T N}(x)$ are monotonic. Both values $x_{T N}$ and $x_{P N}$ are functions of the frequency $\omega$.

This equation describes all the basic features of the structure of Alfvén waves in a dipole-like magnetosphere: logarithmic behavior near the surface of field-line resonance (toroidal surface); the structure of Alfvén wave as an Airy function in the region of mode poloidality (Leonovich and Mazur, 1993; Vetoulis and Chen, 1996); propagation of the wave across the magnetic shells caused by the curved field lines (Leonovich and Mazur, 1993, 1997; Klimushkin et al., 2004). The field lines curvature is taken into account by the finite quantity $\Delta_{N}$. Indeed, in the magnetosphere model with straight field lines the toroidal and poloidal frequencies coincide, i.e. $x_{T N}=x_{P N}$.
Let the functions $\Omega_{T N}(x)$ and $\Omega_{P N}(x)$ be linear (monotonically decreasing) with the similar incline:

$\Omega_{T N}(x)=\Omega_{0}\left(1-\frac{x}{l}\right), \Omega_{P N}(x)=\Omega_{0}\left(1-\frac{x+\Delta_{N}}{l}\right)$.

Then the location of the toroidal surface is determined as $x_{T N}=l\left(\Omega_{0}-\omega\right) / \Omega_{0}$, and the distance between the toroidal and poloidal surfaces $\Delta_{N}=x_{T N}-x_{P N}$ does not depend on the frequency. For the sake of definiteness, we will suppose $\Delta_{N}>0$, though with the finite pressure the opposite situation is possible (Klimushkin et al., 2004). Under these suppositions, the solution of Eq. (1), bounded when $|x| \rightarrow \infty$, can be written in an integral form (Leonovich and Mazur, 1999)

$$
\Phi(x, \omega)=\frac{i q(\omega)}{K} \int_{0}^{\infty} d k F(k)
$$

where

$$
\begin{aligned}
F(k) & =\frac{\exp \left[i K \Delta_{N} h(k)+g(k)\right]}{\sqrt{k^{2}+K^{2}}}, \\
g(k) & =-a k \delta+K a \gamma \arctan \frac{k}{K} \\
h(k) & =\frac{k}{K} \frac{x-x_{T N}}{\Delta_{N}}+\arctan \frac{k}{K}
\end{aligned}
$$

Note that the functions $F, h, g$ also depend on $x$ and $\omega$ as on parameters.

The poloidality condition $\left(\left|\Omega_{T N}-\Omega_{P N}\right| / \Omega_{T N} \gg m^{-1}\right)$ mentioned in Introduction as applied to Eq. (1) can be written in a form $K \Delta_{N} \gg 1$ (Klimushkin et al., 2004). If this condition is satisfied (which is possible only if $m \gg 1$ ), then the integral can be estimated with the method of stationary phase. Omitting intermediate algebraic manipulations, let us show the final expression:

$$
\Phi(x, t)=f(x) \exp \left[i \phi(x)+\Gamma(x)-\frac{i \pi}{4}\right],
$$

where is designated

$$
\begin{aligned}
f(x)= & \frac{i q \sqrt{\pi}}{K^{3 / 2}\left(x_{T N}-x\right)^{1 / 4}\left(x-x_{P N}\right)^{1 / 4}} \\
\phi(x)= & \int_{x_{P N}}^{x} k_{x}\left(x^{\prime}\right) d x^{\prime} \\
= & -K \sqrt{\left(x-x_{P N}\right)\left(x_{T N}-x\right)} \\
& +K \Delta_{N} \arctan \sqrt{\frac{x-x_{P N}}{x_{T N}-x}} \\
\Gamma(x)= & -a \delta \sqrt{\frac{x-x_{P N}}{x_{T N}-x}}+K a \gamma \arctan \sqrt{\frac{x-x_{P N}}{x_{T N}-x}} .
\end{aligned}
$$

This asymptotics is valid at a quite large distance from the poloidal and toroidal surfaces; to determine the asymptotics near $x_{P N}$ and $x_{T N}$, see Leonovich and Mazur (1999).

Expression (3) describes the wave propagating across the magnetic shells. Radial component of the wave vector is

$$
k_{x}=K \sqrt{\frac{x-x_{P N}(\omega)}{x_{T N}(\omega)-x}} .
$$


The mode is localized between the surfaces $x_{T N}$ and $x_{P N}$; we will also call this interval a transparent region because the wave vector radial component squared is positive there. During the propagation, the wave polarization is changed from poloidal to toroidal, as was first found by Leonovich and Mazur (1993). The wave loses energy because of the interaction with the ionosphere and acquires energy because of the interaction with the energetic particles (factor $\Gamma$ in the exponent). On the order of magnitude, the wave amplitude is $|\Phi| \sim q K^{-1}\left(K \Delta_{N}\right)^{-1 / 2}$.

The dependence $k_{x}$ over $\omega$ means the appearance of the radial component of the group velocity:

$$
u_{x}=\left(\frac{\partial k_{x}}{\partial \omega}\right)^{-1}
$$

It is important to notice that $u_{x}$ appeared in MHD regime due to field line curvature (Leonovich and Mazur, 1993), as distinct from the homogeneous plasma case, where the group velocity of Alfvén wave was directed parallel to the ambient magnetic field. Let us determine the time of packet travel from the poloidal surface to the shell with the radial coordinate $x$ :

$$
t_{0}(x, \omega)=\int_{x_{P N}}^{x} \frac{d x^{\prime}}{u_{x}}=\tau \sqrt{\frac{x-x_{P N}}{x_{T N}-x}}
$$

where

$$
\tau=\frac{K l}{\Omega_{0}} .
$$

Asymptotics outside of the transparent region is

$$
\Phi(x, t)=\frac{i q}{K^{2}\left[\left(x-x_{P N}\right)+i a(\delta-\gamma)\right]} .
$$

In the case of the zero field line curvature $\left(\Delta_{N}=\right.$ 0 ), solution (2) describes the localized toroidal resonance (Leonovich and Mazur, 1997).

\section{The Switching on the Source}

Let us consider the oscillations excited by the source with the frequency spectrum $q(\omega)$. Using the Fourier-transform, we obtain from (2) the space-temporal structure of the wave field:

$$
\Phi(x, t)=\frac{i}{2 \pi K} \int_{-\infty}^{\infty} d \omega e^{-i \omega t} q(\omega) \int_{0}^{\infty} d k F(k) .
$$

Later on, using the terms toroidal and poloidal surface, we will determine their position at a frequency $\omega_{0}$, i.e. $x_{T N, P N} \equiv x_{T N, P N}\left(\omega_{0}\right)$.

In this article, the quasi-monochromatic source with a frequency $\omega_{0}$ is considered, switched on the moment of time $t=0$ and switched off after the time interval $T$ :

$$
q(t)=q_{0} e^{-i \omega_{0} t} \Theta(t) \Theta(T-t),
$$

the spectrum of which is

$$
q(\omega)=q_{0} \int_{0}^{T} e^{i\left(\omega-\omega_{0}\right) t} d t
$$

or

$$
q(\omega)=q_{0} \frac{e^{i\left(\omega-\omega_{0}\right) T}-1}{i\left(\omega-\omega_{0}\right)} .
$$

Since we wish to study a passage to the monochromatic regime, we will suppose the period of time $T$ to be rather large, so as $\omega_{0} T \gg 1$.

Taking Eq. (12) into formula (10), we obtain:

$$
\begin{aligned}
\Phi(x, t)= & \frac{i q_{0}}{K} \int_{0}^{\infty} d k F(k) \\
& \cdot \int_{0}^{T} d t^{\prime} \exp \left(-i \omega_{0} t^{\prime}\right) \delta\left[t^{\prime}-\left(t-\frac{k l}{\Omega_{0}}\right)\right],
\end{aligned}
$$

or

$$
\Phi(x, t)=\frac{i q_{0}}{K} \exp \left(-i \omega_{0} t\right) \int_{0}^{t \Omega_{0} / l} d k F(k)
$$

when $0<t<T$ and

$$
\Phi(x, t)=\frac{i q_{0}}{K} \exp \left(-i \omega_{0} t\right) \int_{(t-T) \Omega_{0} / l}^{t \Omega_{0} / l} d k F(k)
$$

with $t>T$.

Let us consider the process of the switching on the source $(t<T)$. We will introduce a new variable

$$
\eta=t / \tau
$$

Further, we will suppose $T \rightarrow \infty$.

\subsection{Initial stage of evolution}

Integral (14) can be estimated by the integration by parts. In this case, the major input into the integral is made by the vicinities of the limits of integration. This asymptotic method cannot be applied if the wave phase has the extreme inside the integrating region. In our case, this is the time instant $t_{0}$, determined by Eq. (8). When $t \ll t_{0}$, we have:

$$
\begin{aligned}
\Phi(x, t) & =\frac{q_{0}}{K^{2}}\left\{\frac{\exp [-i \Psi(x, t)+g(\eta)]}{\sqrt{1+\eta^{2}}\left[\left(x-x_{T N}\right)+\frac{\Delta_{N}}{\left(1+\eta^{2}\right)^{2}}\right]+i a \delta-\frac{i a \gamma}{\left(1+\eta^{2}\right)^{2}}}\right. \\
& \left.-\frac{\exp \left(-i \omega_{0} t\right)}{x-x_{P N}+i a(\delta-\gamma)}\right\},
\end{aligned}
$$

where

$$
\Psi(t, x)=\Omega_{T N}(x) t-K \Delta_{N} \arctan \frac{t}{\tau} .
$$

In expression (16), the value $\Psi$ gives a quantity

$$
\Omega(t, x) \equiv \frac{\partial \Psi}{\partial t},
$$

which can be called "the local instantaneous frequency" of the wave. This quantity is changed from $\Omega_{P N}$ up to $\Omega_{T N}$. This phenomenon is related to the field line curvature and was first described by Leonovich and Mazur (1998).

The role of the instant wave vector is given by the quantity

$$
\tilde{k}_{x}(t, x) \equiv \frac{\partial \Psi}{\partial x} .
$$

When $t \gg \tau$, this value increases in time as $\left|\tilde{k}_{x}\right|=$ $\left|d \Omega_{T N} / d x\right| t$. Accordingly, the radial wavelength decreases, which is clearly seen from Fig. 2. It indicates also that the oscillations change their polarization in time from the 


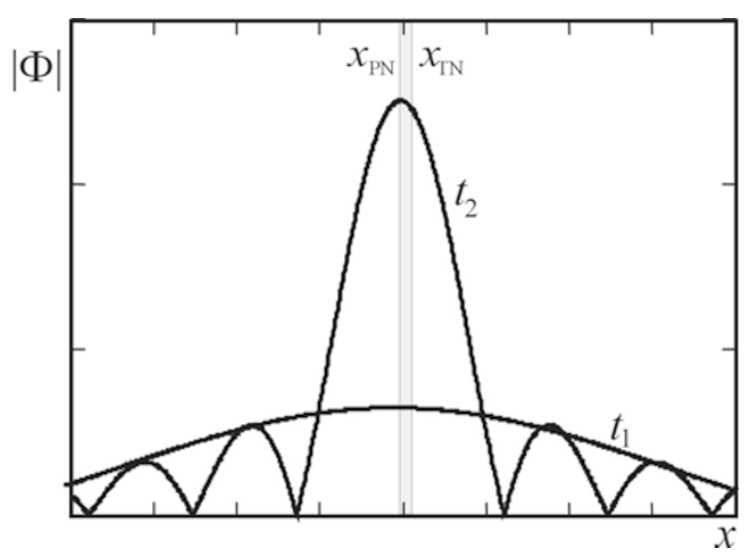

Fig. 2. The spatial structure of the wave field immediately after the source has turned on at two instants of time $\left(t_{2}>t_{1}\right)$. The vertical shaded strip is a transparent region.

poloidal to the toroidal. On the order of the magnitude, the time of the polarization change from the poloidal to the toroidal one is $\tau$.

At the initial stage of evolution, the order-of-magnitude estimate of the wave amplitude is $|\Phi| \sim q K^{-1}\left(K \Delta_{N}\right)^{-1}$. If $K \Delta_{N} \gg 1$, it is much smaller than the amplitude of the monochromatic wave: the wave is only growing.

The denominator of the first term of Eq. (16) is responsible for the temporal change of the wave amplitude. It is easy to see that this value is decreasing, i.e. the wave amplitude increases, as is seen from Fig. 3 obtained by the numerical integrating of Eq. (14). Let us note that in the absence of the field line curvature $\left(\Delta_{N}=0\right)$, the amplitude of this term is decreasing because of the factor $\left[1+(t / \tau)^{2}\right]^{-1 / 2}$ in the first term, which is associated with the phase mixing of the initial disturbance. Here is expressed the significant difference of the waves in the magnetosphere model with the curved field lines from the case of straight lines.

The amplitude also increases due to the instability, which is gradually stabilized with time, because $a \gamma K \arctan (t / \tau) \rightarrow a \gamma K \pi / 2$, as also took place in the work by Klimushkin and Mager (2004). The wave attenuation is associated with the term $\delta$. This factor is related to the energy loss because of the finite conductivity of the ionosphere.

Now, let us consider the very start of the source operation, when $t \ll \tau$, considering also $a \delta$ and $a \gamma$ to be negligibly small as compared with $\Delta_{N}$. Expression (16) then becomes

$$
\Phi(x, t)=\frac{q_{0}}{K^{2}\left[x-x_{P N}\left(\omega_{0}\right)\right]}\left[e^{-i \Omega_{P N}(x) t}-e^{-i \omega_{0} t}\right] .
$$

This formula describes beating with the leading frequency $\left(\Omega_{P N}+\omega_{0}\right) / 2$ and the modulation frequency $\left(\Omega_{P N}-\omega_{0}\right) / 2$. The oscillation with the frequency $\Omega_{P N}$ accounts for the evolution of initial field disturbance, which had appeared at the start of the source operation. Oscillation with a frequency $\omega_{0}$ is conditioned by the continuous operation of a source.

If the denominator of Eq. (16) is turned into zero, the method of integration by parts cannot be used for the asymptotic estimation of the integral. It is easy to see that

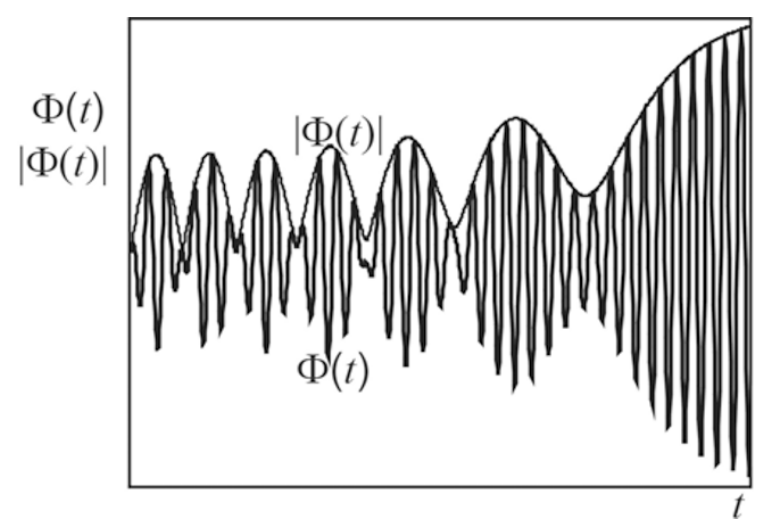

Fig. 3. The temporal behavior of the wave field in a given point of space immediately after the source has turned on (before a wave front arrival).

the turning to zero is performed at the moment of time $t_{0}$ defined by Eq. (8). We will formally consider the asymptotic (16) when $t_{0}-t \ll \tau$ in the region quite distant from the poloidal surface, so that we can neglect the second term of (16):

$\Phi(x, t)=\frac{q_{0} \tau \sqrt{\Delta}}{2 K^{2}} \frac{\exp \left[-i \omega_{0} t+i \phi\left(\omega_{0}, x\right)-\frac{i \alpha}{2}\left(t-t_{0}\right)^{2}\right]}{\left(t_{0}-t\right)\left(x-x_{P N}\right)^{1 / 2}\left(x_{T N}-x\right)}$,

where

$$
\alpha=\frac{\tau^{2} \Delta_{N}}{K}\left[x-x_{P N}\left(\omega_{0}\right)\right]^{-1 / 2}\left[x_{T N}\left(\omega_{0}\right)-x\right]^{-3 / 2},
$$

and $\phi$ was determined by Eq. (7). In yielding (18), we also neglected the attenuation and the instability.

In the next paragraph, we will consider the evolution when $t \simeq t_{0}$ in details.

\subsection{Wave front arrival}

The meaning of the value $t_{0}$ is already known for us: for this time the wave front moving with the radial group velocity arrives at the point with coordinate $x$ from the poloidal surface. It is important to mention that this front if propagating only within the transparent region, since outside of this region $t_{0}^{2}<0$. Hence, outside the transparent region, the evolution is always determined by the phase mixing.

To explore the situation when $t \simeq t_{0}$, we will come back to Eq. (10). The integral over $d k$ will be estimated by the stationary phase method, as in Section 2; thus, here we have Eq. (3). In the expression for $q(\omega)$ (12), we will change the integration variable $t$ for $t^{\prime}$. Putting all this into (10), we obtain:

$$
\Phi(x, t)=\frac{i q_{0}}{2 \pi K} \int_{-\infty}^{\infty} d t^{\prime} q\left(t^{\prime}\right) \int_{-\infty}^{\infty} d \omega e^{-i \omega\left(t-t^{\prime}\right)+i \phi} f e^{\Gamma} .
$$

Because the spectral density of the source is localized near the frequency $\omega_{0}$ in the $T \rightarrow \infty$ case, the major input into the integral is made by the vicinity of this point. To begin, we will decompose the phase $\phi(x, \omega)$ near the point $\omega_{0}$ into power series $\left(\omega-\omega_{0}\right)$ up to a linear term:

$$
\phi(\omega, x)=\phi\left(\omega_{0}, x\right)+\left.\frac{\partial \phi}{\partial \omega}\right|_{\omega_{0}} \cdot\left(\omega-\omega_{0}\right) .
$$




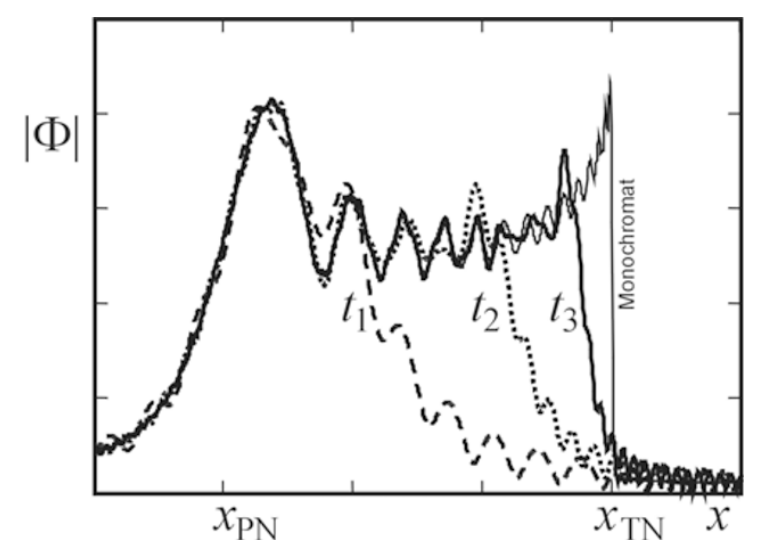

Fig. 4. The wave front movement: wave amplitude $|\Phi(x)|$ at three time instants. The amplitude of the monochromatic wave is also shown.

Note that $\partial \phi\left(\omega_{0}, x\right) / \partial \omega=t_{0}$. Substituting (21) into (20), we obtain after some manipulations:

$$
\Phi=q_{0} f_{0}(x) \Theta\left[t-t_{0}(x)\right] \Theta\left[T-t+t_{0}(x)\right] e^{-i \omega_{0} t+i \phi_{0}(x)+\Gamma_{0}(x)} .
$$

Here the index " 0 " marks the function taken in the point $\omega=\omega_{0}$; for example, $\phi_{0}(x) \equiv \phi\left(\omega_{0}, x\right)$. The expression under the exponent sign coincides with the same one in formula (3). Two Heaviside step functions in (22) describe the leading and back fronts coming through the point $x$ in the transparent region at the moment of time $t_{0}(x)$. As we can see, behind the front, the wave structure is the same as in the monochromatic case: we can say that the front carries the monochromatic regime. In approximation (21), before the front the amplitude is equal to zero; it corresponds to the smallness of the wave amplitude at the initial stage of evolution as compared with the monochromatic wave amplitude.

The more accurate description of the wave front is given by taking into account the next term in the series expansion $\phi(x, \omega)$ :

$$
\phi(\omega, x)=\phi_{0}+\phi_{0}^{\prime}\left(\omega-\omega_{0}\right)+\frac{\phi_{0}^{\prime \prime}}{2}\left(\omega-\omega_{0}\right)^{2},
$$

where

$$
\left.\phi_{0}^{\prime \prime} \equiv \frac{\partial^{2} \phi(x, \omega)}{\partial \omega^{2}}\right|_{\omega_{0}}=\alpha
$$

Here the value $\alpha$ was determined by Eq. (19). After we substitute (23) into (20) and keep in consideration that limit $T \rightarrow \infty$, we obtain the expression

$$
\begin{aligned}
\Phi(x, t)= & \frac{i q_{0}}{2 \pi K} \sqrt{2} \pi f_{0}(x)\left[\frac{1-i}{2}+C\left(z_{1}\right)-i S\left(z_{1}\right)\right] \\
& \cdot e^{-i \omega_{0} t+i \phi_{0}(x)+\Gamma_{0}(x)} .
\end{aligned}
$$

Here the $C$ and $S$ are the Fresnel functions of the argument $z_{1}=\left(t-t_{0}\right) / \sqrt{\pi \phi_{0}^{\prime \prime}}$.

Equation (24) describes the wave front (Fig. 4), which does not have such a clear border as the front described by the Heaviside function (22). Thus, in approximation (23) we obtained the smearing of the wave packet.

When $z_{1} \rightarrow-\infty$, the asymptotic of (24) coincides with (18), that is the approximated solutions at the initial stage of evolution and at the stage of the wave packet arrival are matched with each other.

\subsection{Transition to the monochromatic regime}

When $t \gg t_{0}$ we will use the representation

$$
\Phi(x, t)=-\frac{i q_{0}}{K} e^{-i \omega_{0} t} \int_{0}^{\infty} d k F(k)+\tilde{\Phi},
$$

where

$$
\tilde{\Phi}=\frac{i q_{0}}{K} e^{-i \omega_{0} t} \int_{\frac{i \Omega_{0}}{l}}^{\infty} d k F(k) .
$$

The first term in Eq. (25) describes the monochromatic wave. Thus, after the wave front has passed, we have a superposition of the monochromatic wave and the nonstationary disturbance described by the second item (25), $\tilde{\Phi}$. Through integration by parts we get its estimate:

$$
\begin{aligned}
& \tilde{\Phi}(x, t)=\frac{q_{0}}{K^{2}} \exp \left(-i \omega_{0} t\right) \\
& \cdot \frac{\exp \left[i K\left(x-x_{T N}\right) \eta+i K \Delta_{N} \arctan \eta-a K \delta \eta+a K \gamma \arctan \eta\right]}{\Delta_{N}\left(1+\eta^{2}\right)^{-1 / 2}+\left(x-x_{T N}\right)\left(1+\eta^{2}\right)^{1 / 2}}
\end{aligned}
$$

If the inequality $t \gg \tau$ is also true, then the nonstationary addition to the monochromatic solution has a form

$$
\begin{aligned}
\tilde{\Phi}(x, t)= & -\frac{q_{0}}{K^{2}\left[x-x_{T N}\left(\omega_{0}\right)\right]} \frac{t}{\tau} \exp \left[-i \Omega_{T N} t-a \delta K \frac{t}{\tau}\right. \\
& \left.+i K \Delta_{N} \frac{t}{\tau} a \gamma K \frac{\pi}{2}\right] .
\end{aligned}
$$

On this order of magnitude, the amplitude of this addition is $|\Phi| \sim q_{0} K^{-1}\left(K \Delta_{N}\right)^{-1}$. Because $K \Delta_{N} \gg 1$, then it is much smaller than the amplitude of the monochromatic wave. Moreover, mode (26) is attenuating as $(t / \tau) e^{-a \delta K t / \tau}$. As we can see, even in the case of a very small dissipation, the non-steady disturbance is damping because of the phase mixing.

Notice also that the instability has been stabilized: bounce-drift resonance is not amplifying the wave, the instability has already increased the amplitude $a \gamma K(\pi / 2)$ times. The same situation took place in the case of the zeroduration impulse source (Klimushkin and Mager, 2004).

Thus, after the front has passed, the oscillation becomes the monochromatic wave with a small non-stationary addition with decreasing amplitude.

\subsection{Near the resonance surfaces}

In the vicinity of the poloidal surface, the considerations of the three previous subsections fail because, firstly, solution (16) has a singularity at $x=x_{P N}\left(\omega_{0}\right)$, and, secondary, the time of impulse coming is formally equal to zero. The first circumstance means that immediately after the source has been switched on, the poloidal frequency plays the role of the resonance eigenfrequency.

First, we will consider the evolution near the poloidal surface right after the source has turned on. After evaluation of indeterminate form at $x=x_{P N}\left(\omega_{0}\right)$ in Eq. (17), we have:

$$
\Phi(x, t)=\frac{i q_{0}}{K} \exp \left(-i \omega_{0} t\right) \frac{t}{\tau} .
$$

In this case, the wave amplitude is linearly increasing in time, which is typical for the switching on the source with a 
frequency equal to the eigenfrequency, well known from the general oscillations theory. In that theory, the stabilization due to the non-linear members of the Hamiltonian is taken into account. But in our case the secular increase takes place only during the limited time interval $t \ll \tau$ and it does not lead to the infinite amplitude.

It is quite complicated to explore the evolution at $t \gg \tau$ in the vicinity of the poloidal surface because $t_{0}=0$ there and it is impossible to use the considerations of Subsection 3.2. However, we can use the fact that at $x \simeq x_{P N}$ the major part of integral (2) is accumulated at $k \ll K$ (Leonovich and Mazur, 1999). Then, integral (14) can be represented as

$$
\begin{aligned}
\Phi(x, t)= & \frac{i q_{0}}{K^{2}} \exp \left(-i \omega_{0} t\right) \int_{0}^{t \Omega_{0} / l} d k \\
& \cdot \exp \left[i k\left(x-x_{P N}\right)-i \frac{K \Delta_{N}}{3} \frac{k^{3}}{K^{3}}+g(k)\right]
\end{aligned}
$$

and calculated numerically. The structure of the wave field at several sequential instants $t$ is presented in Fig. 5. We can see here the gradual transmission from the "resonance" structure to the monochromatic wave.

As for the toroidal surface, here the time of monochromatic structure establishing $t_{0}$ is infinite. Consequently, with any suppositions about the work duration of the source $T$, this structure cannot be established there.

\section{Wave Field Evolution after the End of the Source Operation}

Let us consider the case $t>T$, when the field is represented by integral (15). It can be rewritten as

$$
\begin{aligned}
\int_{(t-T) \Omega_{0} / l}^{t \Omega_{0} / l} F(k) d k= & \int_{0}^{\infty} F(k) d k-\int_{0}^{t^{\prime} \Omega_{0} / l} F(k) d k \\
& -\int_{\left(T+t^{\prime}\right) \Omega_{0} / l}^{\infty} F(k) d k
\end{aligned}
$$

where $t^{\prime}=t-T>0$. We will assume that the time of the source operation is long enough, so after the source has turned off the wave field is only a little different from the monochromatic wave field. Thus, the lower and upper limits in the third integral are almost equal $(T / \tau \rightarrow \infty)$, and we can neglect this integral. The first integral describes the monochromatic wave. The second integral is mathematically equal to integral (14), which describes the start of oscillations. Thus, the task has reduced to the one solved in the previous paragraph. The sequence of events after the switching off repeats that had happened after the switching on in the inverse order: first, a comparatively small term is removed from the monochromatic wave field (when $t^{\prime} \ll \tau$ the term has a character of beating, and then the term is increasing with the back front approaching), then through this point comes the wave front, "absorbing" the monochromatic wave and leaving only a small remainder, which is decreasing because of the phase mixing and the dissipation

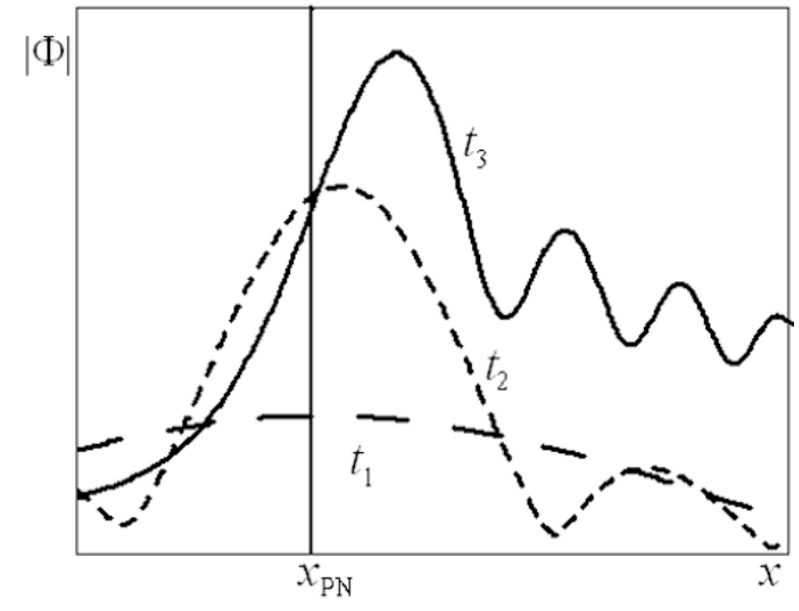

Fig. 5. The spatial structure in the vicinity of the poloidal surface at three instants of time.

on the ionosphere. Let us show the expression for the field at this last stage of evolution (when $t^{\prime} \gg \tau$ ):

$$
\Phi(x, t) \simeq \frac{\tau}{t^{\prime}} \frac{q_{0}}{K^{2}\left(x-x_{T N}\right)} e^{-i \Omega_{T N}(x) t^{\prime}-K a \delta \frac{t^{\prime}}{\tau}} .
$$

The evolution of the wave is accompanied by the increase of the derivative function $\Phi$ with respect to the radial coordinate. It means that the wave gains the toroidal polarization. It is also refers to the vicinity of the poloidal surface, where it had the poloidal polarization at the stage of monochromatic wave. The characteristic time of polarization change is $t^{\prime} \sim \tau$. Thus, we can say that the wave dies being the toroidal one.

\section{Comparison with the Case of the Zero or Small Curvature}

If the source is the delta-function of time, then the differences in the evolution of the wave field in the $\Delta_{N}=0$ case (no curvature) and in the $K \Delta_{N} \gg 1$ case (curvature is essential) are comparatively small, which is obvious from the comparison of the results of the papers of Leonovich and Mazur (1998), Antonova et al. (1999), and Klimushkin and Mager (2004) on the one hand, and, for example, Radoski (1974), Mann and Wright (1995), and Mann et al. (1997) on the other. In our case, when the source has the finite duration, the situation is different markedly from the case of straight field lines, when $\Delta_{N}=0$.

To see it, put $\Delta_{N}=0$ into Eq. (16). At times $t \gg \tau$ the amplitude of the first term is decreasing as $t^{-1}$ even even for negligible small dissipation $(\delta=0)$. It means that just after the source has turned on, the field starts to decrease due to the phase mixing, except for the vicinity of the toroidal resonance surface, where the Alfvén resonance gradually grows; see Wright (1992). In our case $\left(K \Delta_{N} \ll\right.$ $1)$, instead, the amplitude increases because the wave front is approaching. This phenomenon is associated with the curvature.

In our work, we have considered the case of large azimuthal wave numbers, when $K \Delta_{N} \gg 1$. It is instructive also to consider the case $K \Delta_{N} \sim 1$, when $m$ is not so large, and the curvature is still important. In this case, it is easy 


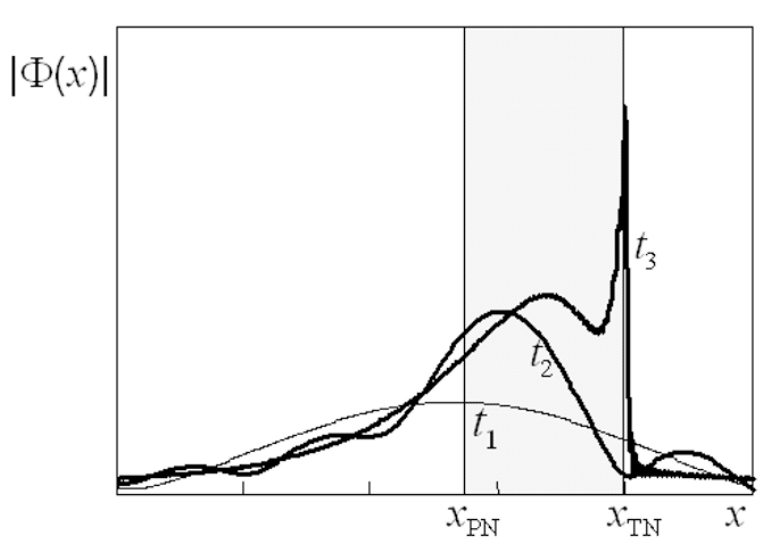

Fig. 6. The evolution of the wave field in the case $K \Delta_{N}=6$.

to take integral (14) numerically. The results are shown in Fig. 6. As it is seen, the initial stage of evolution is not differing much from the case $K \Delta_{N} \gg 1$. But then the fluent increase of the wave amplitude is formed aloof from the toroidal surface. Oscillation structure, typical for the case $K \Delta_{N} \gg 1$, is not appeared.

\section{Conclusion}

In this paper, we have considered the generation of the high- $m$ poloidal Alfvén waves by non-stationary source with finite time duration. We intended to explore how monochromatic poloidal waves appear from initial phasemixing evolution, and how these disappears after the end of the source operation. We took into account the factors that cause the polarization splitting of the spectrum, like field lines curvature and finite plasma pressure.

We gained the following picture (Fig. 7). Immediately after the source has turned on, the wave front appears to move in the radial direction from the poloidal to toroidal surface. At the points where the front has not come yet, the evolution of the wave field is determined by the phase-mixing phenomenon. After the front has passed the given point, the wave amplitude is abruptly increased and the wave structure appears practically similar with the monochromatic one. Outside the transparent region the wave front does not propagate, thus the amplitude is gradually decreased due to the phase mixing there, and localization across $L$-shells is appearing. After the source has turned off, from the poloidal surface there starts moving the back front, which takes away with it the monochromatic wave, leaving a small reminder, which is damping because of the phase mixing and dissipation on the ionosphere.

This picture is markedly different from the $m \sim 1$ case studied by Wright (1992), where there was no analogy of the wave front, and the field starts to decrease just after the source has turned on due to the phase mixing, except for the vicinity of the toroidal surface, where the Alfvén resonance gradually grows.

The wave grows due to the instability when it has poloidal polarization and stops growth when it acquires the toroidal polarization. The increase of amplitude of oscillations with $m \gg 1$ over time has been observed (Fenrich and Samson, 1997) using the SuperDARN HF radar chain.

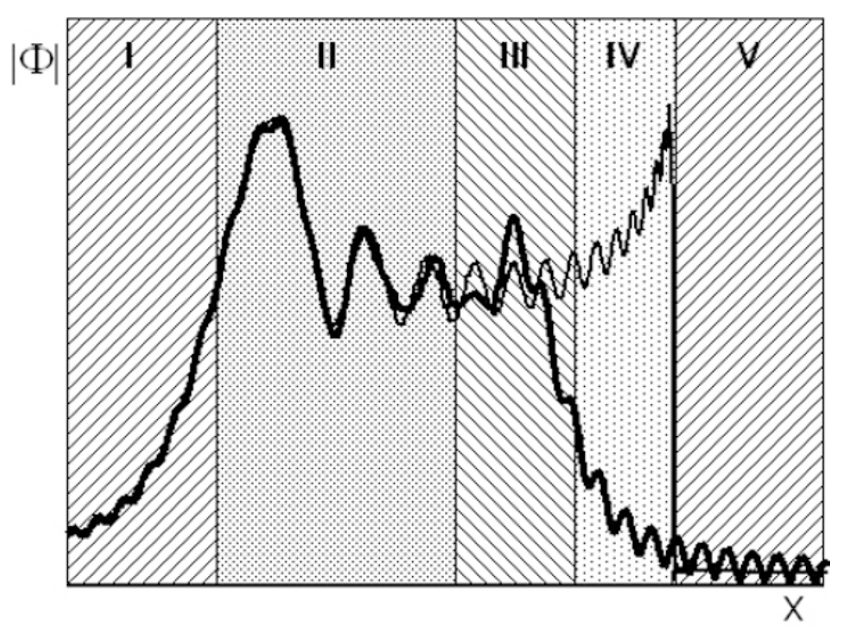

Fig. 7. Spatio-temporal structure of the wave during the time of the source operation: I, V: Phase mixing (out of the transparent region); II: Almost established monochromatic wave; III: The wave front is passing; IV: Phase mixing (the wave front has not passed yet).

Though the authors explained the enhancement of high- $m$ waves in terms of the drift-bounce instability, we have to note that the instability can be imitated by the growth of the wave amplitude when the leading front arrives.

Acknowledgments. The work by D. K. is partially supported by by INTAS grant 05-1000008-7978, by the Russian Foundation for Basic Research (RFBR No 07-05-00950), by Program of presidium of Russian Academy of Sciences \#16 and OFN RAS \#16. J. B. Cao is supported by NSFC Grant 40474062 and the International Collaboration Research Team Program of the Chinese Academy of Sciences.

\section{References}

Anderson, B. J., Statistical studies of Pc 3-5 pulsations and their relevance for possible source mechanisms of ULF waves, Ann. Geophys., 11, 128143, 1993.

Antonova, A. E., Yu. I. Gubar', and A. P. Kropotkin, Towards the model of relativistic electron fluxes: acceleration in the field of strong Alfvénic disturbances, Radiation Measure., 30, 515-521, 1999.

Denton, R. E. and G. Vetoulis, Global poloidal mode, J. Geophys. Res., 103, 6729-6739, 1998.

Denton, R. E., M. R. Lessard, and L. M. Kistler, Radial localization of magnetospheric guided poloidal Pc 4-5 waves, J. Geophys. Res., 108(A3), 1105, doi:10.1029/2002JA009679, 2003.

Dungey J. W., Hydromagnetic waves, in Physics of Geomagnetic Phenomena, Vol. II, 913-934, 1967.

Fenrich, F. R. and J. C. Samson, Growth and decay of field line resonances, J. Geophys. Res., 102, 20,031-20,039, 1997.

Glassmeier, K.-H., ULF pulsations, in Handbook of Atmospheric Electrodynamics, Vol. II, 463-502, 1995.

Hasegawa, A., K. N. Tsui, and A. S. Assis, A theory of long period magnetic pulsations. 3. Local field line oscillations, Geophys. Res. Lett., 10, 765-767, 1983.

Klimushkin, D. Yu., Method of description of the Alfvén and magnetosonic branches of inhomogeneous plasma oscillations, Plasma Phys. Rep., 20, 280-286, 1994.

Klimushkin, D. Yu., Theory of azimuthally small-scale hydromagnetic waves in the axisymmetric magnetosphere with finite plasma pressure, Ann. Geophys., 16, 303-321, 1998a.

Klimushkin, D. Yu., Resonators for hydromagnetic waves in the magnetosphere, J. Geophys. Res., 103, 2369-2378, 1998 b.

Klimushkin, D. Yu., The propagation of high- $m$ Alfvén waves in the Earth's magnetosphere and their interaction with high-energy particles, J. Geophys. Res., 105, 23,303-23,310, 2000.

Klimushkin, D. Yu., How energetic particles construct and destroy poloidal high- $m$ Alfvén waves in the magnetosphere, Planet. Space Sci., 55, 
722-730, 2007.

Klimushkin, D. Yu. and P. N. Mager, The spatio-temporal structure of impulse-generated azimuthally small-scale Alfvén waves interacting with high-energy charged particles in the magnetosphere, Ann. Geophys., 22, 1053-1060, 2004.

Klimushkin, D. Yu., A. S. Leonovich, and V. A. Mazur, On the propagation of transversally-small-scale standing Alfvén waves in a threedimensionally inhomogeneous magnetosphere, J. Geophys. Res., 100, 9527-9534, 1995.

Klimushkin, D. Yu., P. N. Mager, and K.-H. Glassmeier, Toroidal and poloidal Alfvén waves with arbitrary azimuthal wave numbers in a finite pressure plasma in the Earth's magnetosphere, Ann. Geophys., 22, 267$288,2004$.

Leonovich, A. S., Magnetospheric MHD response to a localized disturbance in the magnetosphere, J. Geophys. Res., 105, 2507-2520, 2000.

Leonovich, A. S. and V. A. Mazur, A theory of transverse small-scale standing Alfvén waves in an axially symmetric magnetosphere, Planet. Space Sci., 41, 697-717, 1993.

Leonovich, A. S. and V. A. Mazur, Linear transformation of the standing Alfvén waves in an axisymmetric magnetosphere, Planet. Space Sci., 43, 885-893, 1995a.

Leonovich, A. S. and V. A. Mazur, Magnetospheric resonator for transverse-small-scale standing Alfvén waves, Planet. Space Sci., 43, 881-883, 1995b.

Leonovich, A. S. and V. A. Mazur, A model equation for monochromatic standing Alfvén waves in the axially-symmetric magnetosphere, J. Geophys. Res., 102, 11,443-11,456, 1997.

Leonovich, A. S. and V. A. Mazur, Standing Alfvén waves in an axisymmetric magnetosphere excited by a non-stationary source, Ann. Geophys., 16, 914-920, 1998.

Leonovich, A. S. and V. A. Mazur, Standing Alfvén waves in the magnetosphere from a localized monochromatic source, J. Geophys. Res., 104, 2411-2420, 1999.
Mager, P. N. and D. Yu. Klimushkin, Theory of azimuthally small-scale Alfvén waves in an axisymmetric magnetosphere with small but finite plasma pressure, J. Geophys. Res., 107, 1356, doi:1029/2001JA009137, 2002.

Mager, P. N. and D. Yu. Klimushkin, On impulse excitation of the global poloidal modes in the magnetosphere, Ann. Geophys., 24, 2429-2433, 2006.

Mann, I. R. and A. N. Wright, Finite lifetimes of ideal poloidal Alfvén waves, J. Geophys. Res., 100, 23,677-23,686, 1995.

Mann, I. R., A. W. Wright, and A. W. Hood, Multiple-timescales analysis of ideal poloidal Alfvén waves, J. Geophys. Res., 102, 2381-2390, 1997.

Radoski, H. R., Highly asymmetric MHD resonances. The guided poloidal mode, J. Geophys. Res., 72, 4026-4033, 1967.

Radoski, H. R., A theory of latitude dependent geomagnetic micropulsations: the asymptotic fields, J. Geophys. Res., 79, 595-613, 1974.

Southwood, D. J. and W. J. Hughes, Theory of hydromagnetic waves in the magnetosphere, Space Sci.Rev., 35, 301-366, 1983.

Takahashi, K., Multisatellite studies of ULF waves, Adv. Space Res., 8(910), (9)427-(9)436, 1988.

Vetoulis, G. and L. Chen, Kinetic theory of geomagnetic pulsations, 3, Global analysis of drift Alfvén-ballooning modes, J. Geophys. Res., 101, 15,441-15,456, 1996.

Wright, A. N., Asymptotic and time-dependent solutions of magnetic pulsations in realistic magnetic field geometries, J. Geophys. Res., 97, 6429-6450, 1992.

Yumoto, K., External and internal sources of low-frequency MHD waves in the magnetosphere-a review, J. Geomag. Geoelectr., 40(3), 293$311,1988$.

D. Yu. Klimushkin (e-mail: klimush@iszf.irk.ru), I. Yu. Podshibyakin, and J. B. Cao 